\title{
Editorial
}

\section{Advances in New Signal Processing Techniques for 5G}

\author{
Kan Zheng, ${ }^{1}$ Xianbin Wang, ${ }^{2}$ Periklis Chatzimisios, ${ }^{3}$ and Wei Xiang ${ }^{4}$ \\ ${ }^{1}$ School of Information and Telecommunication Engineering, Beijing University of Posts \& Telecommunications, \\ Beijing 100088, China \\ ${ }^{2}$ Department of Electrical and Computer Engineering, The University of Western Ontario, London, ON, Canada N6A 5B9 \\ ${ }^{3}$ Department of Informatics, Alexander Technological Educational Institute of Thessaloniki (ATEITHE), \\ 57400 Thessaloniki, Greece \\ ${ }^{4}$ College of Science, Technology and Engineering, James Cook University, Cairns, QLD 4870, Australia
}

Correspondence should be addressed to Kan Zheng; zkan@bupt.edu.cn

Received 6 December 2015; Accepted 7 December 2015

Copyright (C) 2016 Kan Zheng et al. This is an open access article distributed under the Creative Commons Attribution License, which permits unrestricted use, distribution, and reproduction in any medium, provided the original work is properly cited.

The evolution of the air interface and radio access network (RAN) to the fifth generation (5G) mobile networks is dependent highly on the advances in new signal processing techniques. These techniques are capable of achieving high capacity gains at the expense of implementation complexity. Meanwhile, the system designs need to be concerned with propagation features of wireless environments. This special issue brings together some of the latest progress in new signal processing techniques for $5 \mathrm{G}$ networks. The variety of approaches presented in the papers demonstrates the possibilities that exist for future developments.

A joint spatial and power multiplexing (JSPM) scheme in massive multiple-input multiple-output (MIMO) systems is proposed in the paper titled "Design of Joint Spatial and Power Domain Multiplexing Scheme for Massive MIMO Systems." This scheme can make use of resources in not only the spatial but also power domain to achieve high multiplexing gains. The paper titled "Transmission Scheme with Limited Channel State Information Feedback for 3D MIMO System" presents a new transmission scheme for the channel state information-reference signal (CSI-RS), where the CSI-RSs including the long-term and short-term ones are precoded with $3 \mathrm{D}$ beamforming vectors. The corresponding limited CSI feedback scheme is also proposed.

Then, in the paper titled "A Simplified Multiband Sampling and Detection Method Based on MWC Structure for mm Wave Communications in 5G Wireless Networks," the authors present a simplified sampling and detection method based on the modulated wideband converter (MWC) structure. It is able to take advantage of beneficial spectrum aliasing to achieve signal sampling at sub-Nyquist rates.

Transmit antenna selection (TAS) is a well-known technique for improving the system performance of MIMO systems. In the paper titled "Low-Complexity Transmit Antenna Selection in Large-Scale Spatial Modulation Systems," two TAS schemes are proposed with the objective of achieving a good trade-off between computational complexity and system performance. In the last paper, that is, "Sum Rate Analysis of MU-MIMO with a 3D MIMO Base Station Exploiting Elevation Features," the authors investigate the sum rate of MU-MIMO systems with a 3D base station (BS) at differing elevations. It is a good starting point to study the sum rate performance for high-rise buildings in modern cities.

\section{Acknowledgments}

The editors would like to extend their gratitude to the anonymous reviewers for the valuable comments suggested, as well as to the authors for responding positively to the questions raised.

Kan Zheng

Xianbin Wang

Periklis Chatzimisios

Wei Xiang 


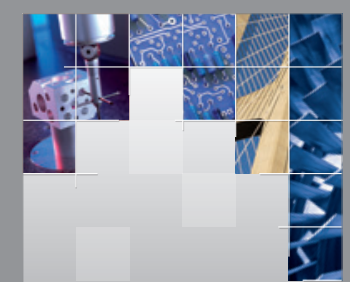

\section{Enfincering}
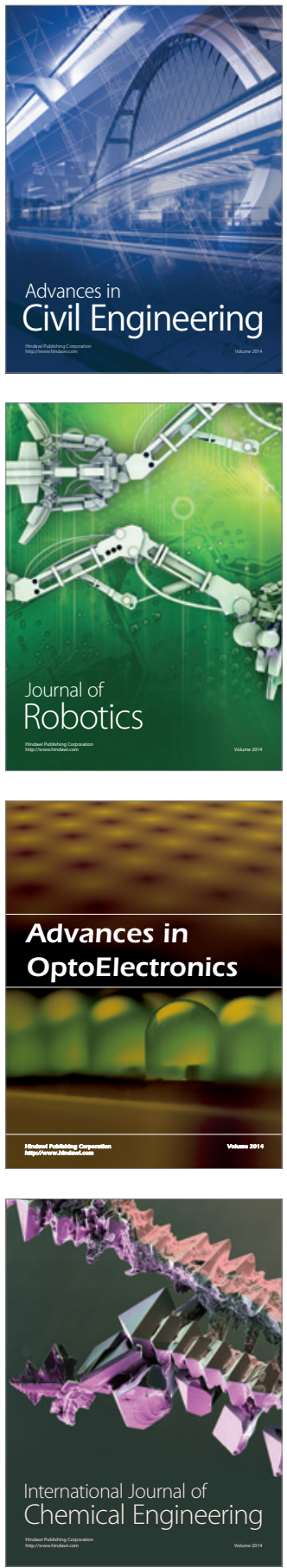

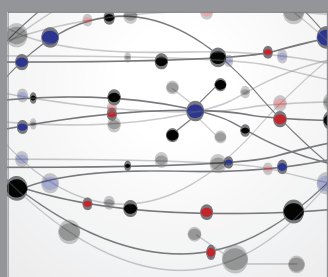

The Scientific World Journal

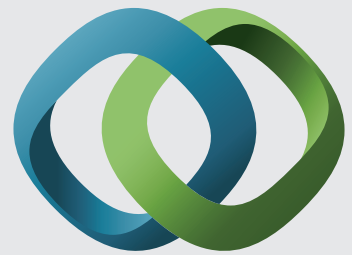

\section{Hindawi}

Submit your manuscripts at

http://www.hindawi.com
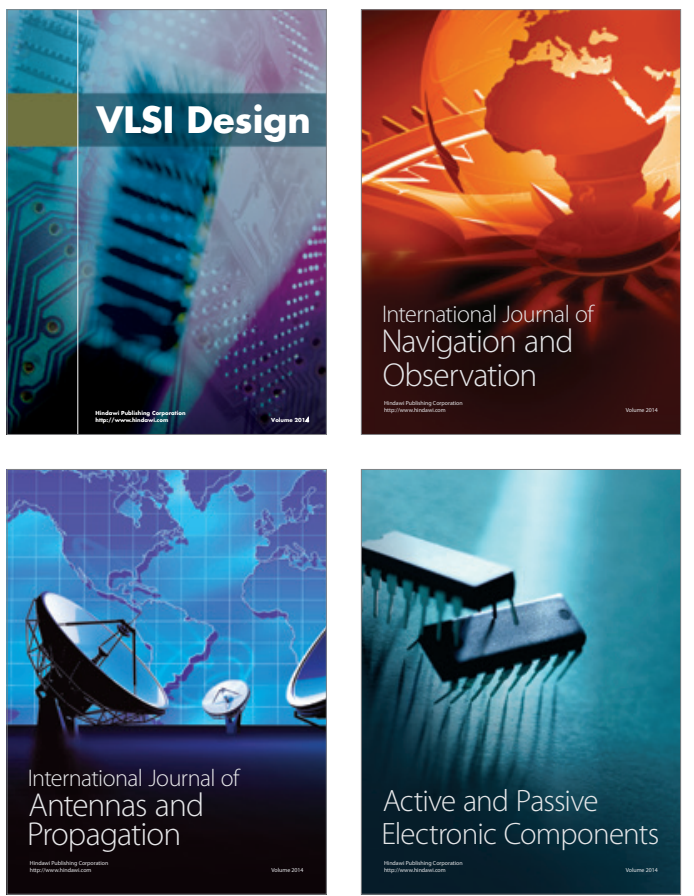
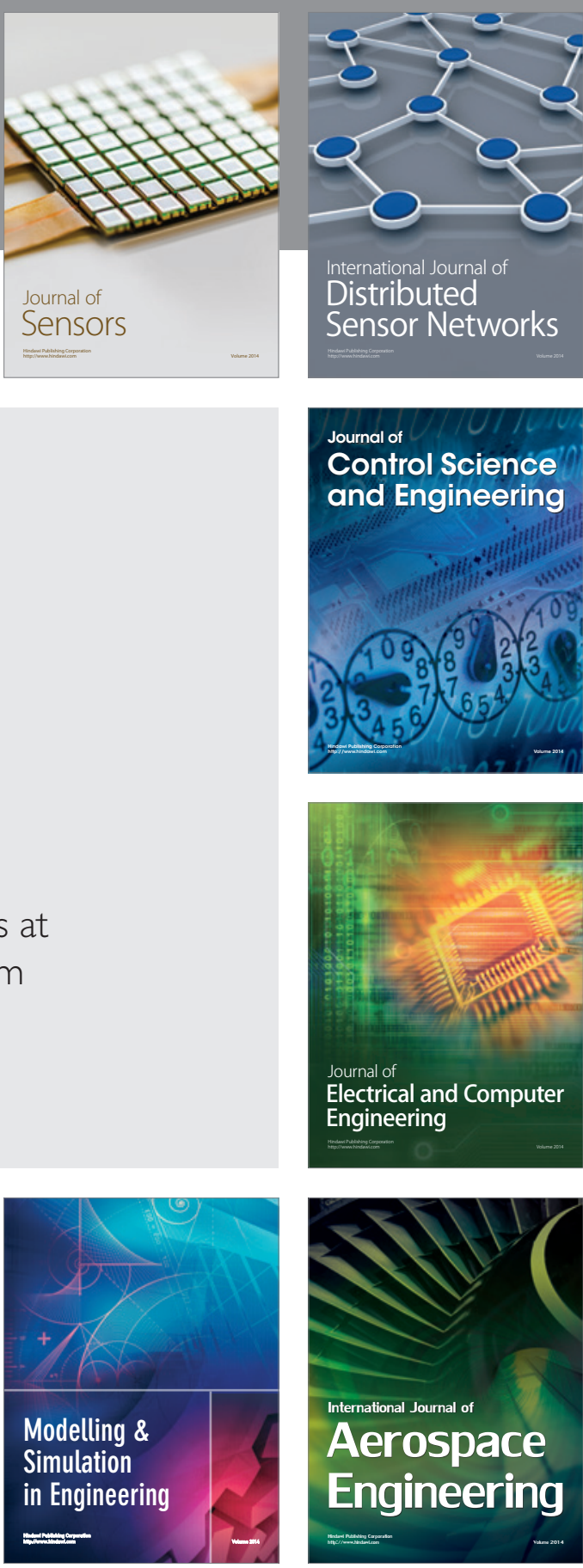

International Journal of

Distributed

Sensor Networks

Journal of

Control Science

and Engineering
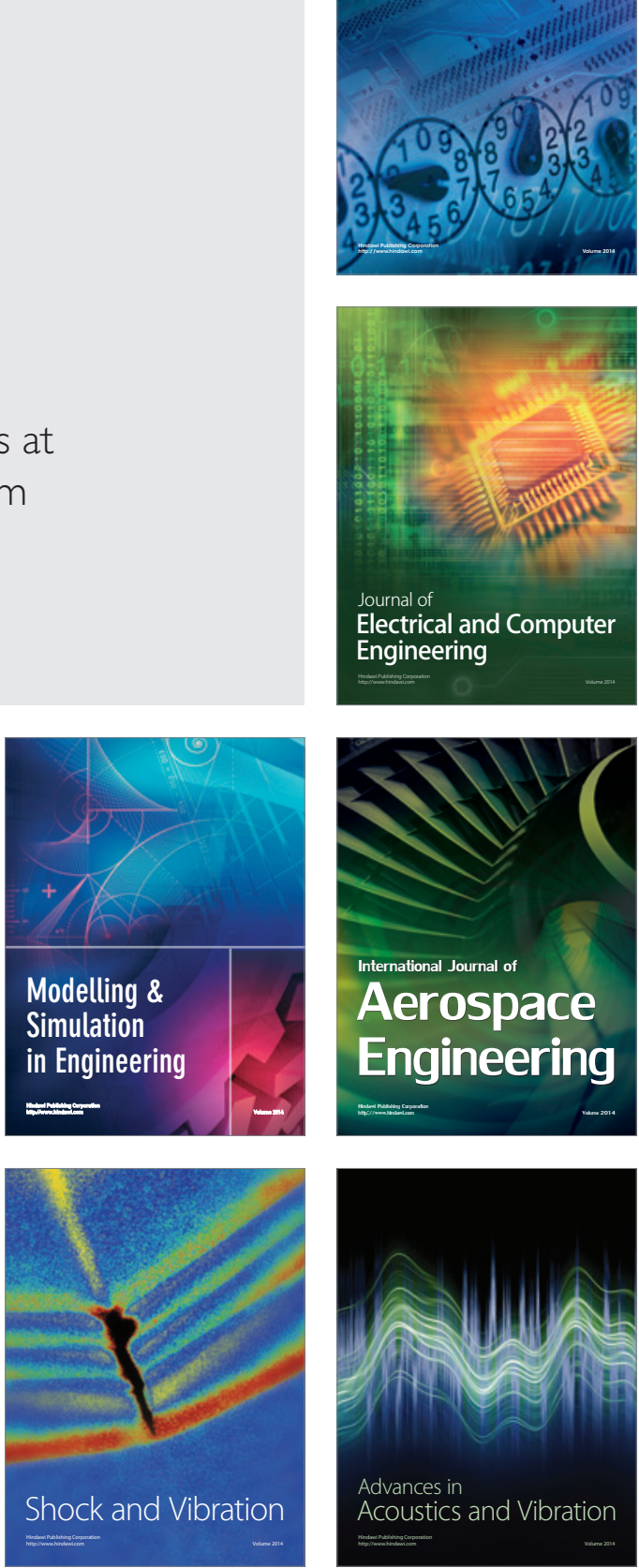